\title{
WEIGHT ENUMERATORS OF SELF-ORTHOGONAL CODES OVER GF(3)*
}

\author{
C. L. MALLOWS $\dagger$ AND N. J. A. SLOANE $\dagger$
}

\begin{abstract}
The Hamming and complete weight enumerators of maximally self-orthogonal codes over $G F(3)$ of lengths $12 m-1,12 m$ and $12 m+1$ are characterized. The results for length $12 m+1$ are believed to be new, while those for length $12 m-1$ and $12 m$ have been considerably simplified.
\end{abstract}

1. Introduction. Professor Marshall Hall, Jr., recently pointed out to us (in connection with his work on the hypothetical projective plane of order twelve) that there is an omission in [6]: the maximally self-orthogonal ternary codes of length $12 m+1$ are not mentioned. An important example of this class is the dual of the code generated by the incidence matrix of the projective plane of order 3 , denoted by $p_{13}$ in [1] and [10]. In fact it is incorrect to say (as we do in [6, p.655]) that if $C$ is a maximally self-orthogonal $\left[n, \frac{1}{2}(n-1)\right]$ code over $G F(3)$ with $1 \in C^{\perp}$ then the extended code $\left(C^{\perp}\right)^{+}$ is self-dual and $n$ is congruent to -1 modulo 12 . We shall see that the correct conclusion is that either $n \equiv-1(\bmod 12)$ and $\left(C^{\perp}\right)^{+}$is self-dual, or $n \equiv+1(\bmod 12)$ and $\left(C^{\perp}\right)^{+}$is not self-dual. The present paper gives the weight enumerators for the missing case. While determining these we were able to considerably simplify the weight enumerators in the case $n \equiv-1(\bmod 12)$ and also when $C$ itself is self-dual and $n \equiv 0(\bmod 12)$. One might say that this is an error-correcting paper.

2. Weight enumerators. Let $C$ be a code of length $n$ and dimension $k$ over $G F(3)$. The complete weight enumerator (cwe) of $C$ is

$$
E_{C}(x, y, z)=\sum_{\mathbf{u} \in C} x^{n_{0}(\mathbf{u})} y^{n_{1}(\mathbf{u})} z^{n_{2}(\mathbf{u})},
$$

where $n_{i}(\mathbf{u})$ is the number of components of $\mathbf{u}$ that are congruent to $i$ modulo 3 . The ordinary or Hamming weight enumerator of $C$ is

$$
W_{C}(x, y)=E_{C}(x, y, y) .
$$

From the MacWilliams theorem the complete weight enumerator of the dual code $C^{\perp}$ is given by

$$
E_{C^{\perp}}(x, y, z)=\frac{1}{3^{k}} E_{C}\left(x+y+z, x+\omega y+\omega^{2} z, x+\omega^{2} y+\omega z\right),
$$

where $\omega=e^{2 \pi i / 3}$ (see [4], [6]).

A code $C$ is called self-orthogonal if $C \subseteq C^{\perp}$ and self-dual if $C=C^{\perp}$. The maximum dimension of a self-orthogonal code of length $n$ is $\frac{1}{2} n$ if $n \equiv 0(\bmod 4), \frac{1}{2}(n-2)$ if $n \equiv 2(\bmod 4)$, and $\frac{1}{2}(n-1)$ if $n$ is odd (see [8], [9]). We wish to characterize the Hamming and complete weight enumerators of self-orthogonal codes of maximal dimension. However our method will only work when we can express the cwe of $C^{\perp}$ in terms of the cwe of $C$. There are two general cases when we can do this:

(i) when $C$ is self-dual, so that

$$
E_{C^{\perp}}(x, y, z)=E_{C}(x, y, z),
$$

\footnotetext{
* Received by the editors October 27, 1980.

$\dagger$ Bell Laboratories, Murray Hill, New Jersey 07974.
} 
(ii) when $C$ is maximally self-orthogonal of odd length and the all-ones vector $\mathbf{1}$ is in $C^{\perp}$ but not in $C$.

In case (ii) we have

$$
\operatorname{dim} C^{\perp}=\operatorname{dim} C+1=\frac{1}{2}(n+1),
$$

which implies

$$
C^{\perp}=C \cup(\mathbf{1}+C) \cup(\mathbf{2}+C)
$$

and

$$
E_{C^{\perp}}(x, y, z)=E_{C}(x, y, z)+E_{C}(y, z, x)+E_{C}(z, x, y)
$$

(since the cwe of $\mathbf{1}+C$ is $E_{C}(y, z, x)$, etc.).

In case (i) the length must be a multiple of 4 , and if we make the additional assumption that the all-ones vector is in $C$ then $n \equiv 0(\bmod 12)$. Without this assumption the results are far more complicated (see [6]). In case (ii) it is a consequence of Theorem 3 below that $n \equiv \pm 1(\bmod 12)$. If $n \equiv-1$ we can add an overall parity check to $C^{\perp}$ so as to make $C^{\perp}$ self-dual, but if $n \equiv+1(\bmod 12)$ this is impossible.

In order to describe the weight enumerators of these codes we introduce the following polynomials. We apologize for the length of this, but it is essential for our method that we work with homogeneous polynomials in six variables. As far as possible we use the same notation as [6].

First the polynomials in $x, y, z$ :

$$
\begin{aligned}
a & =x^{3}+y^{3}+z^{3} \\
f & =x^{2} y+y^{2} z+z^{2} x \\
g & =x y^{2}+y z^{2}+z x^{2} \\
p & =3 x y z \\
\psi_{4} & =x\left(x^{3}+8 y^{3}\right) \\
\phi_{4} & =y\left(x^{3}-y^{3}\right) \\
\xi_{4} & =x\left(y^{3}-z^{3}\right) \\
b & =x^{3} y^{3}+y^{3} z^{3}+z^{3} x^{3} \\
\beta_{6} & =a^{2}-12 b \\
& =x^{6}+y^{6}+z^{6}-10\left(x^{3} y^{3}+y^{3} z^{3}+z^{3} x^{3}\right), \\
v_{7} & =x\left(2 x^{6}-7 y^{6}-7 z^{6}+7 x^{3} y^{3}+7 x^{3} z^{3}-56 y^{3} z^{3}\right), \\
\pi_{9} & =\left(x^{3}-y^{3}\right)\left(y^{3}-z^{3}\right)\left(z^{3}-x^{3}\right) \\
\alpha_{12} & =a\left(a^{3}+8 p^{3}\right) \\
& =\sum^{(3)} x^{12}+4 \sum^{(6)} x^{9} y^{3}+6 \sum^{(3)} x^{6} y^{6}+228 \sum^{(3)} x^{6} y^{3} z^{3}, \\
\tau_{13} & =x y^{6}\left(x^{3}-y^{3}\right)\left(2 x^{3}+y^{3}\right)
\end{aligned}
$$


The second set are polynomials in $u, v, w, x, y, z$ :

$$
\begin{aligned}
\Lambda_{2}= & u x+v y+w z \\
\Xi_{5}= & u x\left(y^{3}-z^{3}\right)+v y\left(z^{3}-x^{3}\right)+w z\left(x^{3}-y^{3}\right), \\
\Upsilon_{8}= & u x\left(2 x^{6}-7 y^{6}-7 z^{6}+7 x^{3} y^{3}+7 x^{3} z^{3}-56 y^{3} z^{3}\right) \\
& +v y\left(2 y^{6}-7 z^{6}-7 x^{6}+7 y^{3} z^{3}+7 x^{3} y^{3}-56 x^{3} z^{3}\right) \\
& +w z\left(2 z^{6}-7 x^{6}-7 y^{6}+7 x^{3} z^{3}+7 y^{3} z^{3}-56 x^{3} y^{3}\right)
\end{aligned}
$$

Note that

$$
\begin{aligned}
& \pi_{9}=g^{3}-f^{3}, \\
& 243 \tau_{13}=x \psi_{4}^{3}-37 x \phi_{4}^{3}-\left.\frac{1}{2} \beta_{6} v_{7}\right|_{y=z}, \\
& \xi_{4}=\Xi_{5}(u=1, v=w=0) \\
& v_{7}=\Upsilon_{8}(u=1, v=w=0)
\end{aligned}
$$

We can now state our results.

THEOREM 1 (Complete weight enumerator). If $C=C^{\perp}$ and $\mathbf{1} \in C$, then $n \equiv 0$ $(\bmod 12)$ and

$$
E_{C}(x, y, z) \in R \oplus \beta_{6} \pi_{9}^{2} R,
$$

where

$$
R=\mathbf{C}\left[\beta_{6}^{2}, \alpha_{12}, \pi_{9}^{4}\right]
$$

In other words the cwe of $C$ can be written uniquely as a polynomial in $\beta_{6}^{2}, \alpha_{12}$ and $\pi_{9}^{4}$, plus $\beta_{6} \pi_{9}^{2}$ times another such polynomial.

COROLlARY 2 (Hamming weight enumerator-Gleason [3]). With the same hypotheses as Theorem 1,

$$
W_{C}(x, y) \in \mathbf{C}\left[\psi_{4}^{3}, \phi_{4}^{3}\right]
$$

TheOREM 3 (Complete weight enumerator). If $C \subset C^{\perp}, \mathbf{1} \in C^{\perp} \backslash C$, and $\operatorname{dim} C^{\perp}=$ $\operatorname{dim} C+1$ then $n \equiv \pm 1(\bmod 12)$.

(a) If $n=12 m+1$ then

$$
E_{C}(x, y, z) \in x R \oplus \beta_{6} v_{7} R \oplus \xi_{4} \pi_{9} R \oplus v_{7} \pi_{9}^{2} R \oplus x \beta_{6} \pi_{9}^{2} R \oplus \xi_{4} \beta_{6} \pi_{9}^{3} R,
$$

where $R$ is defined in Theorem 1.

(b) If $n=12 m-1$ then

(8) $\quad E_{C}(x, y, z) \in \bar{\alpha}_{12} R \oplus \bar{\beta}_{6} \beta_{6} R \oplus \bar{\beta}_{6} \pi_{9}^{2} R \oplus \beta_{6} \bar{\pi}_{9} \pi_{9} R \oplus \bar{\pi}_{9} \pi_{9}^{3} \oplus \beta_{6} \pi_{9}^{2} \bar{\alpha}_{12} R$,

where the bar denotes partial differentiation with respect to $x$.

COROLLARY 4 (Hamming weight enumerator). With the same hypotheses as Theorem 3,

(a) if $n=12 m+1$ then

$$
W_{C}(x, y) \in x \mathbf{C}\left[\psi_{4}^{3}, \phi_{4}^{3}\right] \oplus \tau_{13} \mathbf{C}\left[\psi_{4}^{3}, \phi_{4}^{3}\right],
$$

and

(b) if $n=12 m-1$ then

$$
W_{C}(x, y) \in \bar{\psi}_{4} \psi_{4}^{2} \mathbf{C}\left[\psi_{4}^{3}, \phi_{4}^{3}\right] \oplus \bar{\phi}_{4} \phi_{4}^{2} \mathbf{C}\left[\psi_{4}^{3}, \phi_{4}^{3}\right] .
$$


Extremal weight enumerators. Let us consider the extremal weight enumerators (as defined in [5] and [7]) corresponding to Theorem 3(a) and Corollary 4(a). The first nontrivial iength is 13 , and for simplicity we begin with the Hamming weight enumerator. By Corollary 4(a) the Hamming weight enumerator of any $[13,6]$ self-orthogonal code (with 1 in the dual code) has the form

$$
W(x, y)=c_{1} x \psi_{4}^{3}+c_{2} x \phi_{4}^{3}+c_{3} \tau_{13}
$$

for appropriate constants $c_{1}, c_{2}, c_{3}$. Suppose these constants are chosen so that the minimum weight of the corresponding code (if there is one) is as large as possible. We obtain

$$
\begin{aligned}
W(x, y) & =x \psi_{4}^{3}-24 x \phi_{4}^{3}-132 \tau_{13} \\
& =x^{13}+572 x^{4} y^{9}+156 x y^{12} \\
& =W^{*} \quad \text { (say). }
\end{aligned}
$$

Since $W^{*}$ has nonnegative integral coefficients, it could indeed be the weight enumerator of some $[13,6]$ code $C^{*}$ with minimum weight 9 . On the other hand from Theorem $3(\mathrm{a})$ the complete weight enumerator of $C^{*}$ has the form

$$
E_{C^{*}}(x, y, z)=b_{1} x \beta_{6}^{2}+b_{2} x \alpha_{12}+b_{3} \beta_{6} v_{7}+b_{4} \xi_{4} \pi_{9}
$$

for appropriate constants $b_{i}$. The condition that $C^{*}$ has minimum weight 9 determines the $b_{i}$ uniquely and we find

$$
\begin{aligned}
E_{C^{*}}(x, y, z)=x^{13} & +286 x^{4}\left(y^{6} z^{3}+y^{3} z^{6}\right) \\
& -\frac{13}{9} x\left(y^{12}+z^{12}\right)+\frac{286}{9} x\left(y^{9} z^{3}+y^{3} z^{9}\right)+\frac{286}{3} x y^{6} z^{6} .
\end{aligned}
$$

Since this does not have nonnegative integral coefficients, $C^{*}$ does not exist.

In this case we already knew from the enumeration in [1] that the highest minimum weight attainable is 6 , and furthermore that there is a unique code with minimum weight 6 , namely the projective plane code $p_{13}$ mentioned in $\$ 1$. For this code

$$
\begin{aligned}
W_{p_{13}}(x, y) & =x^{13}+156 x^{7} y^{6}+494 x^{4} y^{9}+78 x y^{12} \\
& =x \psi_{4}^{3}-24 x \phi_{4}^{3}-54 \tau_{13},
\end{aligned}
$$

and

$$
\begin{aligned}
E_{p_{13}}(x, y, z) & =x^{13}+156 x^{7} y^{3} z^{3}+13 x^{4}\left(y^{9}+18 y^{6} z^{3}+18 y^{3} z^{6}+z^{9}\right)+78 x y^{6} z^{6} \\
& =\frac{5}{72} x \beta_{6}^{2}+\frac{17}{24} x \alpha_{12}+\frac{1}{9} \beta_{6} v_{7}+3 \xi_{4} \pi_{9}
\end{aligned}
$$

(which are of the forms (11) and (12)). Although the extremal weight enumerators did not tell us anything new in this example, it is nevertheless interesting to find a situation where the cwe leads to a contradiction not apparent from the Hamming weight enumerator.

For codes of greater length the extremal cwe will probably always contain a negative coefficient (compare [5] and [7]).

Relationship with [6]. The basis for cwe's given in Theorem 1 is simpler than that given in [6, Thm. 1]. The old basis is expressed in terms of the new one by

$$
\gamma_{18}=-\frac{1}{2} \beta_{6}^{3}+\frac{3}{2} \beta_{6} \alpha_{12}+216 \pi_{9}^{2}
$$


and

$$
\begin{aligned}
256 \delta_{36}=-\beta_{6}^{6} & +6 \beta_{6}^{4} \alpha_{12}-9 \beta_{6}^{2} \alpha_{12}^{2}+4 \alpha_{12}^{3} \\
& +864 \beta_{6}^{3} \pi_{9}^{2}-2592 \beta_{6} \pi_{9}^{2} \alpha_{12}-186624 \pi_{9}^{4}
\end{aligned}
$$

The syzygy $\gamma_{18}^{2}=\alpha_{12}^{3}-64 \delta_{36}$ has been replaced by the trivial identity

$$
\left(\beta_{6} \pi_{9}^{2}\right)^{2}=\beta_{6}^{2} \cdot \pi_{9}^{4}
$$

3. The proofs.

Proof of Theorem 1. Since this is parallel to the proofs given in [11] and [12] our treatment will be brief. Suppose $C$ is a self-dual code of length $n=12 m$ containing 1 . Let $G$ be the subgroup of $G L(3, \mathbf{C})$ of order 2952 generated by the matrices

$$
M=\frac{1}{\sqrt{3}}\left[\begin{array}{ccc}
1 & 1 & 1 \\
1 & \omega & \omega^{2} \\
1 & \omega^{2} & \omega
\end{array}\right], \quad J=\left[\begin{array}{ccc}
1 & & \\
& \omega & \\
& & 1
\end{array}\right],
$$

and all $3 \times 3$ permutation matrices. The matrices in $G$ are listed below, in the proof of Lemma 6. In [11] and [12] it is shown that the cwe $E_{C}(x, y, z)$ is invariant under $G$. If $a_{n}$ denotes the number of linearly independent homogeneous invariants for $\mathrm{G}$ of degree $n$, it is also shown in [11] and [12] that the Molien series $\sum_{n=0}^{\infty} a_{n} \lambda^{n}$ is equal to

$$
\frac{1+\lambda^{24}}{\left(1-\lambda^{12}\right)^{2}\left(1-\lambda^{36}\right)}
$$

To find a basis for the invariants we proceed as follows. Let $s=e^{2 \pi i / 12}$. Under the action of $M$ we have

$$
\begin{gathered}
\beta_{6} \stackrel{M}{\rightarrow}-\beta_{6}, \quad \alpha_{12} \stackrel{M}{\rightarrow} \alpha_{12}, \quad x^{3}-y^{3} \stackrel{M}{\rightarrow} s^{11}\left(f-\omega^{2} g\right) \stackrel{M}{\rightarrow}-\left(z^{3}-x^{3}\right), \\
y^{3}-z^{3} \stackrel{M}{\rightarrow} s^{3}(f-g) \stackrel{M}{\rightarrow}-\left(y^{3}-z^{3}\right), \quad z^{3}-x^{3} \stackrel{M}{\rightarrow} s^{7}(f-\omega g) \stackrel{M}{\rightarrow}-\left(x^{3}-y^{3}\right) .
\end{gathered}
$$

Therefore

$$
\pi_{9}=\left(x^{3}-y^{3}\right)\left(y^{3}-z^{3}\right)\left(z^{3}-x^{3}\right)=g^{3}-f^{3} \stackrel{M}{\rightarrow} i \pi_{9}
$$

All of $\beta_{6}, \pi_{9}$ and $\alpha_{12}$ are invariant under $J$, and the permutations fix $\beta_{6}$ and $\alpha_{12}$ and send $\pi_{9}$ to $\pm \pi_{9}$. Thus $\beta_{6}^{2}, \alpha_{12}, \beta_{6} \pi_{9}^{2}$ and $\pi_{9}^{4}$ are indeed invariant under $G$. It only remains to show that $\beta_{6}^{2}, \alpha_{12}$ and $\pi_{9}^{4}$ (or equivalently $\beta_{6}, \alpha_{12}$ and $\pi_{9}$ ) are algebraically independent. This is verified by computing the Jacobian of $\beta_{6}, \alpha_{12}$ and $\pi_{9}$, which is

$$
-2592 x^{2} y^{2} z^{20}+\cdots \text {. }
$$

Since this does not vanish, the polynomials are indeed algebraically independent [2, Thm. 2.3]. Therefore the ring of invariants of $G$ has the form shown on the right-hand side of (5). This completes the proof of Theorem 1.

Proof of Corollary 2. We set $y=z$ in Theorem 1, making $\pi_{9}$ vanish, and then replace $\beta_{6}^{2}$ and $\alpha_{12}$ by the equivalent but simpler pair $\psi_{4}^{3}$ and $\phi_{4}^{3}$.

Proof of Theorem 3. Suppose $C$ is an $\left[n, \frac{1}{2}(n-1)\right]$ self-orthogonal code with $1 \in C^{\perp} \backslash C$. This implies that the cwe of $C$ is a polynomial in $x, y^{3}$ and $z^{3}$, and also satisfies 
(4). From the MacWilliams identity (1) we have

$$
\begin{aligned}
M \circ E_{C}(x, y, z) & =3^{-n / 2} E_{C}\left(x+y+z, x+\omega y+\omega^{2} y, x+\omega^{2} y+\omega z\right) \\
& =3^{-1 / 2} E_{C^{\perp}}(x, y, z) \\
& =3^{-1 / 2}\left\{E_{C}(x, y, z)+E_{C}(y, z, x)+E_{C}(z, x, y)\right\},
\end{aligned}
$$

using (4). Also

$$
\begin{aligned}
M \circ E_{1+C}(x, y, z) & =M \circ E_{C}(y, z, x) \\
& =3^{-n / 2} E_{C}\left(x+\omega y+\omega^{2} z, x+\omega^{2} y+\omega z, x+y+z\right) \\
& =3^{-1 / 2} E_{C^{\perp}}\left(x, \omega y, \omega^{2} z\right) \quad(\text { from }(16)) \\
& =3^{-1 / 2}\left\{E_{C}\left(x, \omega y, \omega^{2} z\right)+E_{C}\left(\omega y, \omega^{2} z, x\right)+E_{C}\left(\omega^{2} z, x, \omega y\right)\right\} \\
& =3^{-1 / 2}\left\{E_{C}(x, y, z)+\omega^{n} E_{C}(y, z, x)+\omega^{2 n} E_{C}(z, x, y)\right\},
\end{aligned}
$$

where in the last step we used the fact that $n_{0}(\mathbf{u}) \equiv n(\bmod 3)$ and $n_{1}(\mathbf{u}) \equiv n_{2}(\mathbf{u}) \equiv 0$ $(\bmod 3)$ for all $\mathbf{u} \in C$. Similarly,

$$
M \circ E_{C}(z, x, y)=3^{-1 / 2}\left\{E_{C}(x, y, z)+\omega^{2 n} E_{C}(y, z, x)+\omega^{n} E_{C}(z, x, y)\right\} .
$$

Since $n$ is odd we have to consider the possibilities $n \equiv \pm 1, \pm 3$ and $\pm 5(\bmod 12)$.

Case 1. $n \equiv \pm 3(\bmod 12)$. The last expressions in (16) and (17) are now identical, implying $E_{C}(x, y, z)=E_{C}(y, z, x)$. Since $C$ always contains $\mathbf{0}$, this implies that $\mathbf{1} \in C$, a contradiction. Thus $n \neq \equiv \pm 3(\bmod 12)$.

Case 2. $n \equiv+1$ or $-5(\bmod 12)$. Now $(17)$ becomes

$$
M \circ E_{C}(y, z, x)=3^{-1 / 2}\left\{E_{C}(x, y, z)+\omega E_{C}(y, z, x)+\omega^{2} E_{C}(z, x, y)\right\} .
$$

We introduce new indeterminates $u, v, w$ and define

$$
F(u, v, w, x, y, z)=u E_{C}(x, y, z)+v E_{C}(y, z, x)+w E_{C}(z, x, y) .
$$

Let $G^{*}$ denote the group of $6 \times 6$ matrices

$$
\left\{A^{*}=\left(\begin{array}{cc}
\bar{A} & 0 \\
0 & A
\end{array}\right) ; A \in G\right\}
$$

of order 2592, where $\bar{A}$ acts on the variables $u, v, w$ and $A$ acts on $x, y, z$. From (16), (17) and (18) it follows that $F$ is invariant under $M^{*}$ and thus under all of $G^{*}$. (Compare the proof of $[6, \mathrm{Thm} .9]$.) We indicate this by writing

$$
F(\bar{A} \mathbf{u}, A \mathbf{x})=F(\mathbf{u}, \mathbf{x}) \quad \text { all } A \in G .
$$

At this point we need the following analogue of $[6$, Thm. 8]. (The proof is essentially the same and is omitted.)

THEOREM 5. Let $G$ be any finite subgroup of $G L(m, \mathbf{C})$, and let $\Phi_{d}$ denote the set of all polynomials $F(\mathbf{u}, \mathbf{x})=F\left(u_{1}, \cdots, u_{m}, x_{1}, \cdots, x_{m}\right)$ which are

(i) homogeneous of total degree $d$,

(ii) linear in the $u_{i}$, and

(iii) satisfy (19).

Let $a_{d}$ denote the number of linearly independent polynomials in $\Phi_{d}$. Then a generating function for the numbers $a_{d}$ is

$$
\sum_{d=0}^{\infty} a_{d} \lambda^{d}=\frac{\lambda}{|G|} \sum_{a \in G} \frac{\operatorname{tr}(\bar{A})}{\operatorname{det}(I-\lambda A)} .
$$


The next step is to compute the sum on the right-hand side of (20) for our group.

LEMMA 6. If $G$ is the subgroup of $G L(3, \mathbf{C})$ of order 2592 defined at the beginning of this section,

$$
\begin{aligned}
\frac{\lambda}{|G|} \sum_{A \in G} \frac{\operatorname{tr}(\bar{A})}{\operatorname{det}(I-\lambda A)} & =\lambda \cdot \frac{\lambda+\lambda^{13}}{\left(1-\lambda^{12}\right)^{3}} \\
& =\lambda \cdot \frac{\lambda+2 \lambda^{13}+2 \lambda^{25}+\lambda^{37}}{\left(1-\lambda^{12}\right)^{2}\left(1-\lambda^{36}\right)} .
\end{aligned}
$$

Proof. We know from [11] and [12] that there are (I) 1944 elements of $G$ of the form

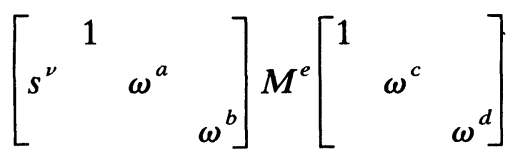

and (II) 648 elements of the form

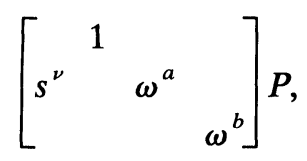

where $0 \leqq \nu \leqq 11,0 \leqq a, b, c, d \leqq 2, e=1$ or 3 and $P$ is any $3 \times 3$ permutation matrix. Instead of $(20)$ we shall work out

$$
\frac{1}{\lambda} \sum_{A \in G} \frac{\operatorname{tr}(\bar{A})}{\operatorname{det}(I-\lambda A)}=\sum_{A \in G} \frac{\operatorname{tr}(\lambda A)^{-1}}{\operatorname{det}(I-\lambda A)}
$$

(since $G$ is a unitary group). Our strategy is to keep $\nu=0$ as long as possible, finally summing over $\nu$ by replacing $\lambda$ by $\lambda s^{\nu}$ and adding. For type (I) we can ignore $c$ and $d$ (and just multiply the final sum by 9) since $c$ can be combined with $a$ and $d$ with $b$ in both $\operatorname{tr}(\lambda A)^{-1}$ and $\operatorname{det}(I-\lambda A)$. The sum on $a, b$ and $e$ is equal to

$$
\begin{aligned}
\frac{2}{1-\lambda^{4}} & +\frac{2 \sqrt{3} / \lambda+6^{*}+\sqrt{3} \lambda+3 \lambda^{2}}{1+\lambda^{6}}+\frac{2 \sqrt{3} / \lambda+4^{*}+2 \sqrt{3} \lambda^{3}+2 \lambda^{4}}{\left(1+\lambda^{2}+\lambda^{4}\right)\left(1-\lambda^{2}+\lambda^{4}\right)} \\
& +\frac{2 \sqrt{3} / \lambda+6^{*}+\sqrt{3} \lambda-3 \lambda^{2}-\sqrt{3} \lambda^{3}-3 \lambda^{4^{*}}-3 \sqrt{3} \lambda^{5}-3 \lambda^{6 *}}{\left(1-\lambda^{2}+\lambda^{4}\right)\left(1+\lambda^{6}\right)} .
\end{aligned}
$$

We replace $\lambda$ by $\lambda s^{\nu}$ and sum over $\nu$; to do this we put everything over powers of $1-\lambda^{12}$ and ignore all terms that are not powers of $\lambda^{12}$ (those not marked with an asterisk). This gives

$$
\frac{24}{1-\lambda^{12}}+\frac{72}{1-\lambda^{12}}+\frac{48}{1-\lambda^{12}}+\frac{12\left(6+6 \lambda^{12}+6 \lambda^{12}+6 \lambda^{12}\right)}{\left(1-\lambda^{12}\right)^{2}}
$$

and multipling by 9 to account for the summation over $c$ and $d$ we find that the contribution from the type (I) terms is

$$
\frac{9 \cdot 144}{1-\lambda^{12}}+\frac{108\left(6+8 \lambda^{12}\right)}{\left(1-\lambda^{12}\right)^{2}}
$$


Next we consider the type (II) terms. When $P=I$ the sum on $a$ and $b$ gives

$$
\frac{9}{\lambda} \frac{1+2 \lambda}{(1-\lambda)\left(1-\lambda^{3}\right)^{2}}
$$

For

$$
P=\left[\begin{array}{lll}
1 & 0 & 0 \\
0 & 0 & 1 \\
0 & 1 & 0
\end{array}\right]
$$

the contribution is

$$
\frac{9}{\lambda} \frac{1}{(1-\lambda)\left(1-\lambda^{6}\right)}
$$

and for

$$
P=\left[\begin{array}{lll}
0 & 1 & 0 \\
1 & 0 & 0 \\
0 & 0 & 1
\end{array}\right] \text { or }\left[\begin{array}{lll}
0 & 0 & 1 \\
0 & 1 & 0 \\
1 & 0 & 0
\end{array}\right]
$$

we get

$$
\frac{9}{\left(1-\lambda^{3}\right)\left(1-\lambda^{6}\right)} \quad \text { (twice) }
$$

Finally

$$
P=\left[\begin{array}{lll}
0 & 1 & 0 \\
0 & 0 & 1 \\
1 & 0 & 0
\end{array}\right] \text { and }\left[\begin{array}{lll}
0 & 0 & 1 \\
1 & 0 & 0 \\
0 & 1 & 0
\end{array}\right]
$$

have trace 0. Adding (23)-(25) we obtain

$$
\frac{9}{\lambda} \frac{2+4 \lambda-2 \lambda^{2}+2 \lambda^{4}}{(1-\lambda)\left(1-\lambda^{3}\right)\left(1-\lambda^{6}\right)}
$$

and we sum over $\nu$ as before, by multiplying top and bottom by $\left(1+\lambda+\lambda^{2}\right)\left(1+\lambda^{3}\right)^{2}(1+$ $\left.\lambda^{6}\right)^{3}$, to get

$$
\frac{9 \cdot 12}{\left(1-\lambda^{12}\right)^{3}}\left(6+36 \lambda^{12}+6 \lambda^{24}\right) \text {. }
$$

The grand total is the sum of (22) and (26):

$$
2596 \frac{1+\lambda^{12}}{\left(1-\lambda^{12}\right)^{3}} \text {. }
$$

We multiply by $\lambda^{2} / 2596$ to obtain (21). The final step is to multiply the top and bottom by $1+\lambda^{12}+\lambda^{24}$ to make the denominator agree with that of (15). This completes the proof of Lemma 6.

Since only exponents of the form $12 m+1$ appear in (21), we see that $n$ cannot be of the form $12 m-5$. It remains to find a basis for the sets $\Phi_{d}$ defined in Theorem 5 . We compute

$$
\Xi_{5} \stackrel{M}{\rightarrow}-\Xi_{5}, \quad \Upsilon_{8} \stackrel{M}{\rightarrow}-\Upsilon_{8}
$$

and deduce the following theorem from (21). 
THEOREM 7. The solutions of (19) that are linear in $u, v, w$ belong to

$$
\Lambda_{2} R \oplus \beta_{6} Y_{8} R \oplus \Xi_{5} \pi_{9} R \oplus Y_{8} \pi_{9}^{2} R \oplus \Lambda_{2} \beta_{6} \pi_{9}^{2} R \oplus \Xi_{5} \beta_{6} \pi_{9}^{3} R .
$$

Finally, setting $u=1$ and $v=w=0$ in Theorem 7, we obtain (7) and thus prove Theorem 3(a).

Case 3. $n \equiv-1$ or $+5(\bmod 12)$. Now $F(u, v, w, x, y, z)$ is invariant under

$$
G^{* *}=\left\{\left(\begin{array}{cc}
A & 0 \\
0 & A
\end{array}\right) ; A \in G\right\} \text {. }
$$

The proof in this case is essentially given in [6, p. 657].

This completes the proof of theorem 3 .

Proof of Corollary 4. We set $y=z$ in Theorem 3, making $\xi_{4}$ and $\pi_{9}$ vanish, and replace $\beta_{6} v_{7}$ by $\tau_{13}$.

Remark. The Taylor series expansion of $(21)$ is

$$
\lambda \sum_{m=0}^{\infty}(m+1)^{2} \lambda^{12 m+1}
$$

Therefore the number of linearly independent homogeneous polynomials of degree $12 m+1$ in the right-hand side of $(7)$ is $(m+1)^{2}$. Similarly the number of degree $12 m-1$ in $(8)$ is $m(m+1)$.

Acknowledgments. We are grateful to Professor Marshall Hall Jr. for pointing out the omission in [6] which led to this work. During this investigation we have made use of two computer programs for symbolic manipulation: the MACSYMA system at the Massachusetts Institute of Technology Laboratory for Computer Science, and the ALTRAN system at the Bell Laboratories Murray Hill Computation Center.

\section{REFERENCES}

[1] J. H. Conway, V. Pless And N. J. A. SloAne, Self-dual codes over GF(3) and GF(4) of length not exceeding 16, IEEE Trans. Information Theory, IT-25 (1979), pp. 312-322.

[2] L. FlatTo, Invariants of finite reflection groups, L'Enseignement mathématique, 24 (1978), pp. 237-292.

[3] A. M. Gleason, Weight polynomials of self-dual codes and the MacWilliams identities, in Actes, Congrès International des Mathématiciens 1970, Vol. 3, Gauthiers-Villars, Paris, 1971, pp. 211-215.

[4] F. J. MacWilliams And N. J. A. Sloane, The Theory of Error-Correcting Codes, North-Holland, Amsterdam and Elsevier/North-Holland, New York, 1977.

[5] C. L. MAllows, A. M. OdLyzKo AND N. J. A. SloAne, Upper bounds for modular forms, lattices and codes, J. Algebra, 36 (1975), pp. 68-76.

[6] C. L. Mallows, V. Pless ANd N. J. A. SloAne, Self-dual codes over GF(3), SIAM J. Applied Math., 31 (1976), pp. 649-666.

[7] C. L. Mallows And N. J. A. Sloane, An upper bound for self-dual codes, Inform. and Control, 22 (1973), pp. 188-200.

[8] V. PLESS, The number of isotropic subspaces in a finite geometry, Rend. Cl. Scienze Fisiche, Matematiche e Naturali, Acc. Naz. Lincei, 39 (1969), pp. 418-421.

[9] - On the uniqueness of the Golay codes, J. Combinatorial Theory, 5 (1968), pp. 215-228.

[10] V. Pless, N. J. A. Sloane And H. N. WARd, Ternary codes of minimum weight 6 and the classification of the self-dual codes of length 20, IEEE Trans. Information Theory, IT-26 (1980), pp. 305-316.

[11] N. J. A. SloANE, Weight enumerators of codes, in Combinatorics, M. Hall Jr. and J. H. van Lint, eds., Reidel, Dordrecht and Mathematical Centre, Amsterdam, 1974, pp. 115-142.

[12] N. J. A. SLOANE, Error-correcting codes and invariant theory: new applications of a nineteenth-century technique, Amer. Math. Monthly, 84 (1977), pp. 82-107. 